\title{
Self-consistent calculations for shallow defects in semiconductors: II. Donor-acceptor pairs
}

\begin{abstract}
A M Stoneham and A H Harker
Theoretical Physics Division, Atomic Energy Research Establishment, Harwell, Oxfordshire, OX11 0RA.

Received 11 November 1974

Abstract. A general method has been developed for performing self-consistent calculations for shallow defects or defect complexes in semiconductors. Results are given here for donoracceptor pairs in GaP and a comaprison is made of the various treatments of central-cell corrections and the effects arising from the overlap of the donor and acceptor wavefunctions.

Whilst no detailed refitting of host parameters is attempted, it seems likely that the current estimate of the bandgap $(2.339 \mathrm{eV})$ is too large, and the latest estimates of the dielectric constant may also be inaccurate.
\end{abstract}

\section{Introduction}

One of the classes of defect central to the development of commercial electro-optic devices consists of donor-acceptor pair systems (eg Dean 1973). In these systems, an electron trapped at a donor may recombine with a hole trapped at an acceptor, giving luminescence with an energy $E_{\mathrm{LUM}}$ approaching that of the bandgap, $E_{\mathrm{GAP}}$.

The donor-acceptor pairs are of great scientific interest, in addition to their technological virtues. Extremely accurate spectroscopy is possible, giving recombination energies and other details of the transitions as a function of the donor-acceptor separation. The theory of the trends with separation of the transition energy, $E_{L_{U M}}$, is the subject of the present paper. We shall demonstrate the use of the general computer program SEMELE (Stoneham and Harker 1975, to be referred to as I), comparing earlier treatments of the theory with a more accurate approach and commenting on the implications for the parameters of the host lattice.

\section{General}

In this section we outline previous work on donor-acceptor systems, the choice of suitable systems to test different aspects of the theory and the values of parameters for the various systems studied.

\subsection{Earlier theories}

The results which have been the mainstay of the interpretation of donor-acceptor recombination data are given by Hopfield (1964), whose 'quantum chemistry' approach to shallow defects is followed in the present paper. The essence of his conclusions is that 
at large separations $R$, where the donor and acceptor wavefunctions do not overlap

$$
\Delta E \equiv E_{\mathrm{GAP}}-E_{\mathrm{LUM}} \simeq E_{\mathrm{A}}+E_{\mathrm{D}}-e^{2} / \epsilon R
$$

where $E_{\mathrm{D}}$ is the donor ionization energy, $E_{\mathrm{A}}$ the acceptor ionization energy and $\epsilon$ the dielectric constant (usually taken as the static constant). At very small separations, where the Coulomb fields of the ionized donor and acceptor would cancel to a great extent, Hopfield argues:

$$
\Delta E \simeq E_{\mathrm{EXC}}
$$

where $E_{\mathrm{EXC}}$ is the exciton binding energy.

We shall be concerned primarily with $\Delta E(R)$, together with the effects of central-cell corrections on the various energies. We shall not be discussing the many small corrections to (2.1) proposed by other workers. These include multipole corrections, elastic interactions, polaron effects, van der Waals terms and corrections from the wavevector dependence of the dielectric constant. However, it is worth stressing that the van der Waals terms are not important. The original discussion of this contribution (Trumbore and Thomas 1965) was based on empirical arguments. Revised estimates of the dielectric constant reduced the need for these terms. Further, quantitative estimates are possible (Stoneham 1975) based on the observed polarizabilities of donors and acceptors (Dean and Patrick 1970), which show that the van der Waals terms must be only a few per cent of the original estimates. The problem has been complicated by the incorrect screening used by many workers (including some otherwise-scrupulous analyses, for example, Vink et al 1973): the van der Waals term should contain an $\epsilon^{-2}$ factor, not the $\epsilon^{-1}$ commonly found (McLachlan 1965, Israelachvili 1972). In essence, the instantaneous dipole on one centre induces a dipole proportional to $\epsilon^{-1}$ on the other and the interaction of these two dipoles is screened by a further $\epsilon^{-1}$ factor.

There have been four earlier calculations of the separation dependence of $\Delta E$ which go beyond (2.1). The earliest, due to Williams (1960) took into account the overlap of the donor and acceptor wavefunctions. This work was extended later by Mehrkam and Williams (1972), whose work we discuss in more detail in $\S 3$. Hopfield (1964) quotes otherwise-unpublished calculations in his review. His work appears to cover many of the aspects treated later by Mehrkam and Williams and his results have the added virtue of going over to (2.2) at small spacings. Bindemann and Unger (1973) adopted a strategy different in detail, using 'floating' wavefunctions centred on sites other than the donor and acceptor. Their method also behaves reasonably at short distances. Calculations for molecular $\mathrm{H}_{2}$ (Hurley 1954, Rosen 1931) suggest the Bindemann-Unger treatment should give rather better energies than the Mehrkam-Williams method.

\subsection{Choice of system}

There are several sets of accurate data for donor-acceptor pair recombination. We shall discuss two systems in GaP: the sulphur-carbon pairs and, to a lesser extent, the sulphurzinc pairs. The host crystal, GaP, is convenient because of the accurate experimental data and because it has a rather low degree of ionicity, so that polaron effects are unlikely to be important. The GaP: $(\mathrm{Zn}, \mathrm{S})$ system has been discussed by Mehrkam and Williams and it is for this reason we describe briefly some calculations in $\S 4$. But the major part of the work $(\S 3)$ is on GaP: $(\mathrm{S}, \mathrm{C})$. The advantages of this system are that the most detailed experimental information is available (Dean 1973, Vink et al 1973) and that the carbon is a very shallow acceptor for which an unsophisticated treatment of central-cell correc- 
tions suffices. The sulphur donor is fairly deep and is suitable for comparing the merits of different treatments of the central-cell terms.

\subsection{Choice of parameters}

We shall need to choose values of the dielectric constant $\epsilon$, the effective masses $m_{\mathrm{e}}^{*}$ and $m_{\mathrm{h}}^{*}$, and the band gap $E_{\mathrm{GAP}}$. In addition to these host lattice properties, the effective radii for the core electrons must be established for a description of the central-cell corrections (cf $\S 3$ of the preceding paper).

We shall normally use the following parameters for GaP:

$$
\left.\begin{array}{ll}
\epsilon=11.02 & \text { (Vink et al 1973), } \\
m_{\mathrm{e}}^{*}=0.33 m_{0} & \text { (I, to fit effective-mass donor energy), } \\
m_{\mathrm{h}}^{*}=0.38 m_{0} & \text { (I, to fit effective-mass acceptor energy), } \\
E_{\mathrm{GAP}}=2.339 \mathrm{eV} \text { (Dean 1973). }
\end{array}\right\}
$$

Different parameters are used occasionally. In special cases, notably $\mathrm{GaP}:(\mathrm{Zn}, \mathrm{S})$, we compare results with earlier work using $\epsilon=10 \cdot 75$, and so we alter the dielectric constant. In other cases, central-cell corrections are included by modifying $m_{\mathrm{e}}^{*}$. We shall also see that our calculations cast doubt on the assumed values of $\epsilon$ and $E_{\mathrm{GAP}}$.

As described in $I$, the donor core can be represented by a nuclear charge $Z=5$ and four electrons in orbitals with wavefunctions proportional to $\exp (-\alpha r)$ and $r \exp \left(-\frac{1}{2} \alpha r\right)$. The parameters $\alpha$ can be varied to give the correct central-cell correction. In I we needed to know only that the $\alpha$ controlled the central-cell correction. Here we need to know the precise relation between $\alpha$ and the donor binding. The results of such calculations are given in figure 1. All choices of basis function and all scalings are as described in I. However, there is one slight difference. For systems with more than one centre, as for a donor-acceptor pair, there are advantages in using a Gaussian basis in calculating the molecular integrals. These advantages are very considerable when more than two centres are involved. The program used has an option for expanding the Slater orbitals chosen as a sum of Gaussians. The approximations introduced are slight if enough Gaussians (typically 3 or 4) are used and arise mainly from the poor description of the cusp in the $1 \mathrm{~s}$ core functions. The results in figure 1 have used an expansion in terms of three Gaussians. The same expansion was used in all subsequent calculations.

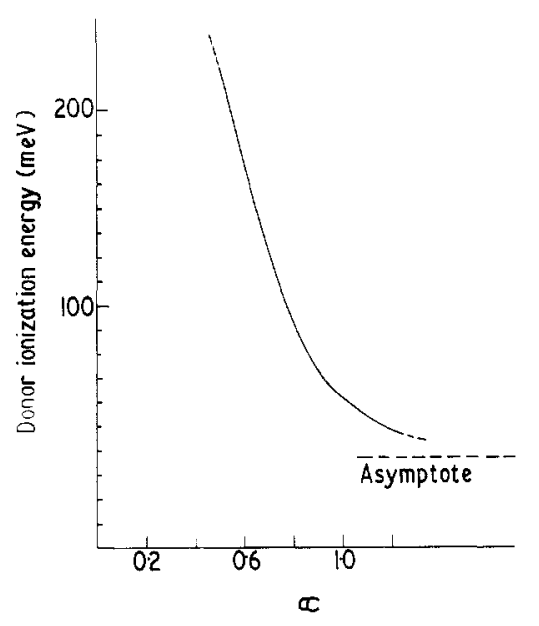

Figure 1. Donor ionization energies in $\mathrm{GaP}$ for various choices of core wavefunctions. These results use $Z=5$, and $1 \mathrm{~s}$ and $2 \mathrm{~s}$ functions $\exp (-x r)$ and $r \exp \left(-\frac{1}{2} \alpha r\right)$ for the four core electrons. This parameterization is appropriate when each Slater function is fitted by three Gaussian functions. The asymptote is for $\alpha \rightarrow \infty$. 


\subsection{Different levels of calculation}

We have calculated the variation of the donor-acceptor pair energy with separation using five different levels of approximation, which we now summarize in $2.4 .1-2.4 .5$. The wavefunction parameters used are listed in table 1 .

Table 1. Parameters used in various calculations for GaP: (S, C)

\begin{tabular}{|c|c|c|c|c|c|}
\hline Method & $\epsilon$ & $m_{\mathrm{e}}^{*} / m_{0}$ & $m_{\mathrm{h}}^{*} / m_{0}$ & Functions & Exponents (au) \\
\hline \multirow[t]{2}{*}{ Mehrkam-Williams } & $11 \cdot 02$ & 0.672 & 0.672 & $1 \mathrm{~s}(\mathrm{D}, \mathrm{A})$ & 0.0609 \\
\hline & & & & $2 \mathrm{p}(\mathrm{D}, \mathrm{A})$ & 0.0609 \\
\hline \multirow[t]{4}{*}{ Asymmetric } & $11 \cdot 02$ & 0.930 & 0.414 & $1 \mathrm{~s}(\mathrm{D})$ & 0.0844 \\
\hline & & & & $1 \mathrm{~s}(\mathrm{~A})$ & 0.0376 \\
\hline & & & & $2 p(D)$ & 0.0844 \\
\hline & & & & $2 \mathrm{p}(\mathrm{A})$ & 0.0276 \\
\hline \multirow[t]{4}{*}{ Extended asymmetric } & $11 \cdot 02$ & 0.930 & $0 \cdot 414$ & $1 \mathrm{~s}, 2 \mathrm{p}(\mathrm{D})$ & 0.0844 \\
\hline & & & & $2 \mathrm{~s}, 2 \mathrm{p}(\mathrm{D})$ & 0.0422 \\
\hline & & & & $1 \mathrm{~s}, 2 \mathrm{p}(\mathrm{A})$ & 0.0376 \\
\hline & & & & $2 \mathrm{~s}, 2 \mathrm{p}(\mathrm{A})$ & 0.0188 \\
\hline \multirow[t]{8}{*}{ Full SEMELE } & 11.02 & 0.33 & 0.414 & is (D) & 0.0842 \\
\hline & & & & $1 \mathrm{~s}, 2 \mathrm{~s}, 2 \mathrm{p}(\mathrm{D})$ & 0.0502 \\
\hline & & & & $2 \mathrm{~s}, 2 \mathrm{p}(\mathrm{D})$ & 0.0421 \\
\hline & & & & $1 \mathrm{~s}, 2 \mathrm{p}(\mathrm{A})$ & 0.0376 \\
\hline & & & & $2 \mathrm{~s}, 2 \mathrm{p}(\mathrm{A})$ & 0.0188 \\
\hline & & 1.0 (core & & 1s (donor core) & 0.75 \\
\hline & & electrons) & & 2s (donor core) & 0.375 \\
\hline & & \multicolumn{4}{|c|}{ Parameters used for $\mathrm{GaP}(\mathrm{Zn}, \mathrm{S})$} \\
\hline Mehrkam-Williams & $10 \cdot 75$ & 0.705 & 0.705 & $1 \mathrm{~s}, 2 \mathrm{p}(\mathrm{D}, \mathrm{A})$ & 0.0581 \\
\hline Extended asymmetric & $10 \cdot 75$ & 0.705 & 0.705 & $1 \mathrm{~s}, 2 \mathrm{p}(\mathrm{D}, \mathrm{A})$ & 0.029 \\
\hline
\end{tabular}

2.4.1. Coulombic form (case I). This form uses equation (2.1) and is asymptotically correct at large distances. But, since donor-acceptor spectra are often used to determine the dielectric constant $\epsilon$, the results may show unduly good agreement with experiment. In particular, one should note that the largest separations for which there are accurate data are only two or three times the effective Bohr radii: the true asymptotic régime and the range of spacings seen in practice do not overlap.

2.4.2. Mehrkam-Williams form (case II). In its most basic version, Mehrkam and Williams (1972) assume a total wavefunction of the form $\psi_{\mathrm{e}}\left(\boldsymbol{r}_{\mathrm{e}}-\boldsymbol{R}_{\mathrm{D}}\right) \psi_{\mathrm{h}}\left(\boldsymbol{r}_{\mathrm{h}}-\boldsymbol{R}_{\mathrm{A}}\right)$, where $\boldsymbol{R}_{\mathrm{D}}$ and $\boldsymbol{R}_{\mathrm{A}}$ give the donor and acceptor positions. The one-particle functions $\psi$ have the form:

$$
\psi(r)=N(1+\sigma \beta Z) \exp (-\beta r)
$$

where $N(\sigma, \beta)$ is a normalization and the $Z$ axis is the axis of the defect. This form of $\psi$ is the simplest which shows most features of interest and it makes several major approximations. First, all asymmetry of donor and acceptor is ignored, even if $E_{\mathrm{D}}$ and $E_{\mathrm{A}}$ differ. The parameters $\sigma$ and $\beta$ are the same for both the electron and hole. Secondly, the centralcell corrections are incorporated by altering the effective masses. Both electron and hole are assumed to have a mass $m^{*}$ given by

$$
\frac{m^{*}}{m_{0}}=\frac{1}{2} \epsilon^{2}\left(E_{\mathrm{A}}+E_{\mathrm{D}}\right)
$$


where $E_{\mathrm{A}}$ and $E_{\mathrm{D}}$ are measured in Rydbergs. This scheme was used in Hopfield's earlier calculations. Thirdly, the exponent $\beta$ was not treated as a full variation parameter. It was fixed by the relation:

$$
\beta=\frac{m_{0}}{m^{*}} \epsilon a_{0} \quad \frac{2 a_{0}}{E_{\mathrm{A}}+E_{\mathrm{D}}}
$$

where $a_{0}$ is the Bohr radius. This value of $\beta$ was held constant for a range of spacings. Note too that the $\beta$ parameter for the 2 p component of $\psi$ is the same as for the $1 \mathrm{~s}$ component; for the hydrogen atom, $\beta_{2 \mathrm{p}}$ is half $\beta_{1 \mathrm{~s}}$. The parameter $\sigma$ is varied freely, altering the weights of 1 s and $2 p$ in the one-electron functions to minimize energy. Mehrkam and Williams call this process 'configuration admixture', a misleading phrase, totally different in meaning to the standard useage of molecular physics (eg Condon and Shortley 1935, Slater 1963, Coulson 1963, McWeeney and Sutcliffe 1969).

2.4.3. Simple asymmetric form (case III). This corresponds to the Mehrkam-Williams model in all respects but one: the asymmetry of donor and acceptor is included. Instead of (2.5), the electron and hole masses are defined by:

$$
\frac{m^{*}}{m_{0}}=\epsilon^{2} E_{\mathbf{D}}
$$

and

$$
\frac{m_{\mathrm{h}}^{*}}{m_{0}}=\epsilon^{2} E_{\mathrm{A}}
$$

where again $E_{\mathrm{A}}$ and $E_{\mathrm{D}}$ are measured in Rydbergs. The orbital exponents for electron and hole are then fixed by the relations:

$$
\beta_{\mathrm{e}}=\frac{m_{0} \epsilon}{m_{\mathrm{e}}^{*}} a_{0}=a_{0} / \epsilon E_{\mathrm{D}}
$$

and

$$
\beta_{\mathrm{h}}=\frac{m_{0} \epsilon}{m_{\mathrm{h}}^{*}} a_{0}=a_{0} / \epsilon E_{\mathrm{A}} .
$$

The asymmetry may be quite considerable. For the GaP: $(\mathrm{S}, \mathrm{C})$ system, for example, $\beta_{\mathrm{e}}$ is only $45 \%$ of $\beta_{\mathrm{h}}$.

2.4.4. Extended basis form (case IV). This is a generalization of case III, but with a more general form of the one-particle functions $\psi$. Instead of only $1 \mathrm{~s}$ and $2 \mathrm{p}$ functions, with exponents $\beta$ defined by (2.9) or (2.10), the following set of functions is used:

Exponent $\beta$ : 1 s and $2 \mathrm{p}$ functions

Exponent $\beta / 2$ : $2 \mathrm{~s}$ and $2 \mathrm{p}$ functions;

that is, four basis functions for the donor and four different ones for the acceptor.

2.4.5. Full central-cell approach (case V). In this form, the central-cell corrections for the donor are included by using a realistic core, as described in $\S 2.3$ of this paper and $\S 3$ of the preceding paper. Additional $2 \mathrm{p}$ basis functions are included, with exponents as for the $2 s$ functions. In the cases we shall be treating, the acceptor is treated as in case IV, namely by a specially-chosen effective mass. This approximation should be reasonable for the shallow carbon acceptor, which we discuss primarily, where the ionization energy is only $46.4 \mathrm{meV}$. 
Table 2. Predictions of various models for $\Delta E$, the difference between the bandgap and the donor-acceptor recombination energy. The difference is in $\mathrm{meV}$ and is positive when the energy of luminescence predicted is less $E_{\mathrm{GAP}}$. Spacings are in atomic units. The singlettriplet splitting uses the full SEMELE method.

\begin{tabular}{lcccccc}
\hline $\begin{array}{l}\text { Spacing } \\
(\mathrm{au})\end{array}$ & Coulombic & $\begin{array}{l}\text { Mehrkam- } \\
\text { Williams }\end{array}$ & Asymmetric & $\begin{array}{l}\text { Extended } \\
\text { asymmetric }\end{array}$ & $\begin{array}{l}\text { Full } \\
\text { SEMELE }\end{array}$ & $\begin{array}{l}\text { Singlet- } \\
\text { triplet } \\
\text { splitting }\end{array}$ \\
\hline 10 & -96.26 & -13.95 & -11.47 & $17 \cdot 23$ & 12.92 & $9 \cdot 22$ \\
20 & $27 \cdot 17$ & 34.80 & 37.05 & 40.41 & 36.79 & $9 \cdot 30$ \\
30 & 68.31 & 65.99 & 67.38 & 67.82 & 65.73 & $9 \cdot 03$ \\
40 & 88.88 & 85.93 & 86.45 & 86.53 & 85.71 & 6.95 \\
60 & 109.46 & 107.48 & 107.45 & 107.46 & 107.96 & $3 \cdot 30$ \\
\hline
\end{tabular}

\section{Results for GaP: (S, C)}

The ionization energies for the two component defects are $104.2 \mathrm{meV}$ (S) and $46.4 \mathrm{meV}$ (C). Results for all five methods and spacings $R=10,20,30,40$ and 60 au are given in table 2 and shown in figure 2. With the parameters adopted, the exciton energy is $8.6 \mathrm{meV}$ (preceding paper) in the open-shell Hartree-Fock procedure used here. This is compatible with the observed values (albeit fortuitously so) and we shall use this value to obtain results for zero spacing. We shall compare the various methods in $\S 5$.

\subsection{Coulomb form}

As mentioned, this is asymptotically exact at large $R$. However, it gives a divergence at small $R$. In the present case (2.1) predicts a transition energy higher than that for the free exciton when $R<R_{0} \sim 9 \cdot 2 \AA$, and results for smaller spacings will surely be unreliable.

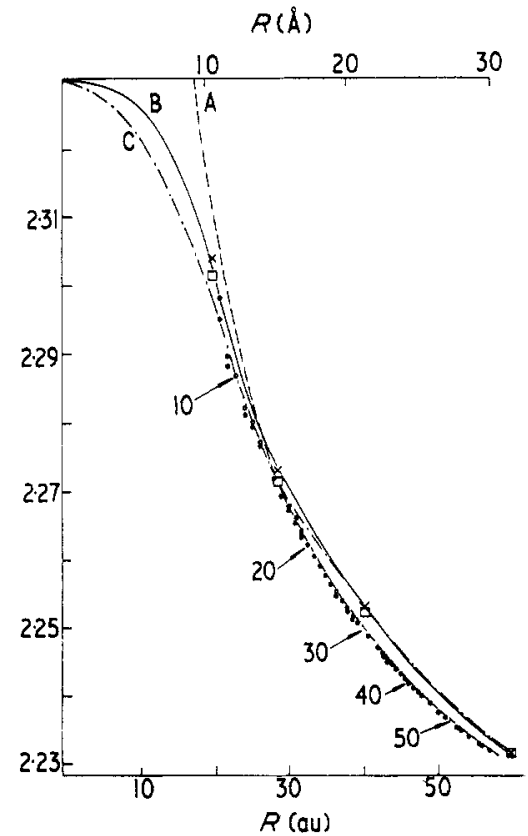

Figure 2. Recombination energies for the $\mathrm{GaP}:(\mathrm{S}, \mathrm{C})$ donor-acceptor pair in various approximations: Curve A, Coulombic method; curve B, full SEMELE; curve $C$, extended asymmetric; the points $\square$, asymmetric; and $\times$, Mehrkam-Williams. The experimental data $(O)$ are taken from Deans (1973). 


\subsection{Mehrkam-Williams form}

This method also becomes unreliable at small $R$, overestimating the transition energy considerably when $R \lessgtr R_{0}$. The reason for the overestimate is the relative inflexibility of the choice of basis. Like all the methods described, this method should converge satisfactorily on the asymptote (2.1) at large spacings. But one important point emerges, relevant for all the methods II-V. The effective Bohr radius is of the order of $8.7 \AA$ and the largest spacings studied are rather less than $30 \AA$. The overlaps are still significant at these distances and it is not trivial to deduce values parameters like $\epsilon$ from the experimental data.

The p-like admixture decreases at large separations and is listed in table 3.

Table 3. $\mathrm{s}-\mathrm{p}$ admixture on the donor in the Mehrkam-Williams and the Asymmetric cases for $\mathrm{GaP}:(\mathrm{S}, \mathrm{C})$. The values quoted are the weights of the normalized $\mathrm{s}$ and $\mathrm{p}$ functions. The charge density between the donor and acceptor is reduced by the admixture. Results for other methods show similar trends, but are more complicated because of the more extended basis used.

\begin{tabular}{llllll}
\hline \multirow{2}{*}{ Method } & \multicolumn{5}{c}{ Separation (au) } \\
\hline Mehrkam-Williams & 0.232 & 20 & 30 & 40 & 60 \\
Asymmetric & 0.421 & 0.246 & 0.120 & 0.067 & 0.0155 \\
\hline
\end{tabular}

\subsection{Simple asymmetry}

As with cases I and II, problems occur for small separations. The general features of II and III agree qualitatively, both as regards energies (table 2) and admixtures of p-like terms (table 3). The admixtures reduce the charge densities in the overlap region. Quantitatively, asymmetry makes a difference of more than $1 \mathrm{meV}$ out to distances of order $20 \AA$ and differences in the donor $\mathrm{p}$-admixture of more than $1 \%$ to similar distances.

\subsection{Extended basis}

This is the simplest method which avoids problems at small spacings. The electron-hole recombination energy appears to converge properly onto the exciton energy at small $R$ and to the asymptote at large $R$.

\subsection{Full central-cell approach}

Like the extended basis method, this form gives the correct asymptotic behaviour at both large and small $R$. However, there is quite a large difference in the values of the transition energy given by this method and the extended basis method at 10 au and 20 au separations. Available experimental data are compatible with both; it is not easy to decide which is the most successful, partly because other complications (eg multipole corrections) are significant.

Our calculations have also been repeated for the spin triplet system, although this has not been observed. The singlet-triplet splittings are listed in table 2 . 


\section{Results for GaP: (Zn, S)}

We have treated this system in much less detail and quote results here primarily to verify the Mehrkam-Williams results and to see the effects of extending the basis used without other sophistications. Consequently, we have used different crystal parameters, namely:

$$
\epsilon=10.75 \quad m_{\mathrm{e}}^{*}=m_{\mathrm{h}}^{*}=0.705 .
$$

The effective masses and exponents $\beta \equiv 0.0581$ au have been chosen using (2.5), (2.6) and the ionization energies of $104 \cdot 2 \mathrm{meV}(\mathrm{S})$ and $64 \mathrm{meV}(\mathrm{Zn})$.

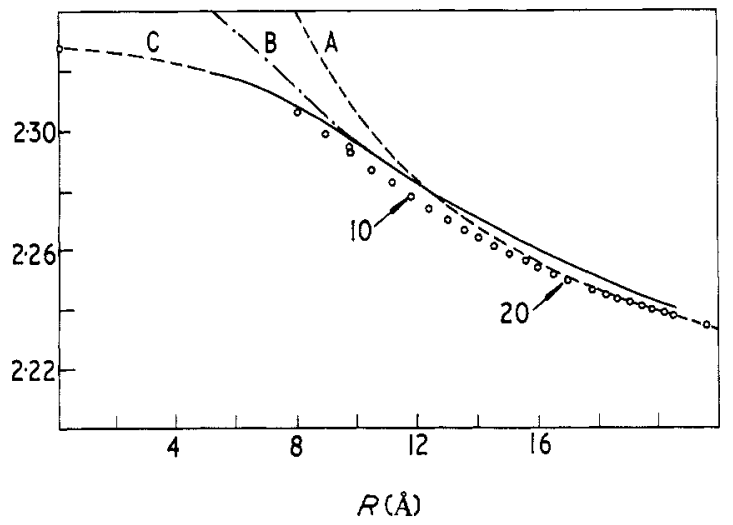

Figure 3. Recombination energies for the GaP: $(\mathrm{Zn}, \mathrm{S})$ donor-acceptor pair in various approximations : Curve A, Coulombic ; curve B, Mehrkam-Williams; and curve C, extended asymmetric. The experimental data $(O)$ are taken from Dean (1973)

The results given in table 4 were obtained with the present program. Mehrkam and Williams give their results in diagrammatic form only; suffice it to say that our data and theirs appear to agree provided one includes a constant shift of their results of the sort described in their paper.

The main difference between the calculations of the Mehrkam-Williams form (case II) and those with a more extended basis (as for case II, but with additional $2 \mathrm{~s}$ and $2 \mathrm{p}$ functions with exponents $\beta / 2$ ) is at small separations. The more extended basis avoids the divergence mentioned in $\$ 3$.

\section{Discussion}

We now compare various aspects of the different approximations, with the aim of deciding which approaches can be used reliably and conveniently in calculations of

Table 4. Predictions of $\Delta E$ in $\mathrm{meV}$ for $\mathrm{GaP}:(\mathrm{S}, \mathrm{Zn})$

\begin{tabular}{lrrrrr}
\hline & \multicolumn{5}{c}{ Separation (au) } \\
Method & \multicolumn{1}{c}{10} & 20 & 24 & \multicolumn{1}{l}{30} & 40 \\
\hline Coulombic & -84.8 & 41.7 & 62.8 & 83.8 & 104.9 \\
Mehrkam-Williams & 0.2 & 46.9 & 60.7 & 77.5 & 97.5 \\
Extended basis & 20.1 & 47.9 & 61.1 & 77.7 & 97.8 \\
\hline
\end{tabular}


donor-acceptor pair spectra. Convenience is important, whilst not an overriding factor. Suffice it to say that we believe the SEMELE program to be particularly straightforward to use; the calculations have been automated, and the program combines simplicity of use with great flexibility.

The first point to discuss is the choice of basis functions. Can one successfully use just a single $\mathrm{s}$ and a single $\mathrm{p}$ function on each site? The answer seems to be no. Both the Mehrkam-Williams and asymmetric approaches behave very badly at small spacings $R$. Indeed, both predict the wrong sign for $\Delta E$ at sinall $R$. But the other approaches (including that of Bindemann and Unger 1973, whose floating functions are equivalent to an expansion in many spherical harmonics with related radial factors) behave properly at small spacings. These successful approaches include the extended basis and full SEMELE methods. We shall return to the results at large spacings later.

The second point concerns the way in which the central-cell corrections are included. Again, the small-separation cases are the strongest tests. There are effects at large spacings but these are masked by the purely Coulombic part of $\Delta E$. Deviations from (2.1) should show a strong model-dependence because of the different asymptotic wavefunctions, but their value is a small fraction of $\Delta E$ at most. The results of table 2 or figure 2 show large differences between the two methods whose behaviour is satisfactory; that is, between the extended basis form and the full SEMELE form. We have not been able to use experiment to decide between these two, partly because the experimental data in the critical 10-20 au range are very incomplete. The problem is complicated further by the need for extra theoretical corrections at small $R$, like multipole corrections. But the full SEMELE form makes more realistic assumptions about the form of the central-cell corrections, so one would expect its predictions to be the most reliable.

The third point concerns the intermediate and larger spacings. At the largest spacings, the Coulombic part of $\Delta E$ (see (2.1)) dominates, and all theories do reasonably well. Indeed, at these spacings it is the parameterizations of the host dielectric constant and bandgap which are the most important factors. The present calculations suggests that the standard parameters adopted $\left(E_{\mathrm{GAP}}=2.339 \mathrm{eV}\right.$ and $\left.\epsilon=11.02\right)$ may be in error. The important point is that the estimates of these parameters are based on the Coulombic form (2.1) at the larger spacings. But the 'large' spacings usually used (to $70 \AA$ for GaP: $(\mathrm{O}, \mathrm{C})$ by Vink et al, but more commonly only $20-30 \AA$ ) are only a few times larger than the effective Bohr radii and overlap terms are still significant. Thus the usual parameters $E_{\mathrm{GAP}}$ and $\epsilon$ are empirical parameters, useful only for interpolation and limited extrapolation, and not correct crystal parameters. It would be a major undertaking to reparameterize the spectra and we have not done this. But the results do suggest that the band gap is 2 to $3 \mathrm{meV}$ smaller than assumed (giving 2.336 to $2.337 \mathrm{eV}$ ). This new value is consistent with all the data with which we are familiar. It also implies a slightly lower exciton binding than usual, about $8 \mathrm{meV}$ instead of $10 \mathrm{meV}$. This too is consistent with other data (see eg Czaja 1971).

Thus, at large spacings, all the approaches work satisfactorily with a proper choice of parameters. The parameters differ significantly between the best fit of the Coulombic form (2.1) and a fit of the methods with overlap corrections. This difference in choice of parameters is also conspicuous at intermediate spacings, where the predictions of $\Delta E$ for all the models with overlap cross the curve for (2.1) at a spacing of two or three effective Bohr radii. Since the crossover appears for all models, it is unlikely to be an artefact and, indeed, one can make plausible arguments for the small- and large- $R$ behaviour which imply a crossover. However, the crossover could be eliminated for practical purposes if the empirical host parameters were used for the Coulombic form (2.1) and correct host 
parameters for the other methods. Further, if the band gap is reduced to $2.337 \mathrm{eV}$, only the full SEMELE approach is satisfactory at smaller $\boldsymbol{R}$.

Differences between the models become apparent at intermediate spacings, where all show trends of the right qualitative form. But, whilst the more advanced methods (extended basis and full SEMELE) give results which lead naturally to the correct small- $R$ behaviour, the two other methods (Mehrkam-Williams and asymmetric) give satisfactory corrections over such a small range of spacings that there is rather little advantage in using them.

\section{Acknowledgments}

We are indebted to Dr V Saunders (Atlas Laboratory) for the copy of ATMOL on which SEMELE is based, and to Drs P J Dean, D C Herbert and A M White (RRE Malvern) for stimulating discussions.

\section{References}

Bindemann R and Unger K 1973 Phys. Stat. Solidi 56(b) 563-71

Condon E U and Shortley G H 1935 Theory of Atomic Spectra (London: Cambridge UP) ch 15

Coulson C A 1961 Valence (London: Oxford UP)

Czaja W 1971 Phys. Kondens. Mater. 12 226-36

Dean P J 1973 Prog. Solid St. Chem. 8 1-126

Dean P J and Patrick L 1970 Phys. Rev. B2 1888-99

Hopfield J J 1964 Proc. Int. Conf. on Semiconductors, Paris pp 725-35

Hurley A C 1954 Proc. R. Soc. 226 179-90

Israelachvili J N 1972 Proc. R. Soc. A331 39-51

McLachlan A D 1965 Discuss, Faraday Soc. 40 238-46

McWeeny R M and Sutcliffe B T 1969 Methods of Molecular Quantum Mechanics (New York: Academic Press)

Mehrkam L and Williams F E 1972 Phys. Rev. B6 3753-6

Rosen N 1931 Phys. Rev. 38 2099-104

Slater J C 1963 Quantum Theory of Molecules and Solids: I (New York: McGraw-Hill) p 45

Stoneham A M 1975 Theory of Defects in Solids (London: Oxford UP)

Stoneham A M and Harker A H 1975 J. Phys. C: Solid St. Phys. $81102-8$

Trumbore F A and Thomas D G 1965 Phys. Rev. 137 A1030-7

Vink A T, van der Heyden R L A and van der Does de Bye J A W 1973 J. Luminescence 8 105-25

Williams F E 1960 J. Phys. Chem. Solids 12 265-73 